\title{
Rapid Immunohistochemistry of resected Bowel Margins at the time of Colectomy: Defining the presence of Premalignant Cells undetected by H\&E
}

\author{
Myron Arlen ${ }^{1,2 *}$, Philip Arlen ${ }^{2}$, Gene Coppa ${ }^{1}$, Jim Crawford ${ }^{1}$, Deutsch $G^{1}$, Procaccino $J^{1}$, Sullivan $J^{1}$, XuePing \\ Wang $^{2}$, Olga Saric ${ }^{2}$, Dubokouvskiy $A^{2}$ and Ernesto Molmenti ${ }^{1}$ \\ 'Division of Surgical Oncology, Department of Pathology, North Shore University Hospital, Northwell Health system, Hosfstra College of \\ Medicine, Manhasset NY, USA \\ ${ }^{2}$ Precision Biologics, Great Neck, NY 11021, USA
}

\section{Article Info}

*Corresponding author: Myrlon Arlen

Division of Surgical Oncology

North Shore University Hospital

Northwell Health system

Manhasset NY

Hosfstra College of Medicine and Precision Biologics

Great Neck NY, USA

E-mail: myronarlen@yahoo.com

Received: May 27, 2017

Accepted: June 8, 2017

Published: June 15, 2017

Citation: Arlen M, Arlen P, Coppa E, et al. Rapid Immunohistochemistry of resected Bowel Margins at the time of Colectomy: Defining the presence of Premalignant Cells undetected by H\&E. Madridge J Vaccines. 2017; 1(1): 9-13.

doi: $10.18689 / \mathrm{mjv}-1000103$

Copyright: (c) 2017 The Author(s). This work is licensed under a Creative Commons Attribution 4.0 International License, which permits unrestricted use, distribution, and reproduction in any medium, provided the original work is properly cited.

Published by Madridge Publishers
Keywords: Immunohistochemistry; Tumor immunogenic protiens; Antibodies; Antigen.

\section{Background and Origin of Target Proteins defined by Immunohistochemistry (IHC)}

Tumor immunogenic proteins (TAA's), specifically associated with colorectal carcinoma, were first isolated from pooled allogeneic membrane preparations derived from colorectal carcinoma operative specimens [1]. They were later characterized in terms of their molecular structure by affinity purification followed by mass spectroscopy. Monoclonal antibodies were subsequently developed against the antigen or antigens so derived [2]. The colon cancer antigens that had been isolated from tumor membrane preparations were then used to immunize BALBC mice for hybridoma production, the antibodies so derived, being screened for specificity. Those that were found to characterize the colon cancer lesion without evidence of cross reactivity to normal colonocytes, were tested clinically for both diagnostic and therapeutic efficacy [3]. They have since, been found capable of functioning to detect the earliest lesions in what appears to be the premalignant state. These same monoclonals were found to be capable of diagnosing the presence of the colon malignancy by both immuno- histochemistry of the biopsied specimens as well as by serum ELISA. Should the tumor have invaded the blood stream, those metastatic lesions resulting from such invasion appear to be effectively targeted by intravenous administration of the monoclonal antibody [4]. When defining the origin of such antigens it was noted that they first became apparent in the early premalignant state of tumor development and remained unchanged as the lesion became invasive. No evidence of mutations were found to develop in the advanced as well as metastatic lesion. As such during a therapeutic approach, when metastasis are noted, it is not necessary to biopsy the metastatic lesion in order to determine which variant of the immunogenic protein is present. Rather we can re: examine the original primary by Immunohistochemistry to determine which therapeutic agent is necessary to control the metastasis.

When Ariel Hollinshead (1985) employed partially purified pooled allogeneic tumor membrane antigen for treating a variety of malignant lesions, it became apparent that when the antigen was delivered to the host at threshold levels and specifically for the malignancy expressing innate antigen, i.e.colon cancer, that the immune system could be shifted from performing immune-surveillance to one of providing a therapeutic mechanism for attacking and destroying the tumor [5].

To define the nature of the tumor protein or proteins capable of inducing enhancement in tumor recognition, the monoclonals that were developed in BALBC mice were studied in 
detail. Three of the antibodies obtained from the fusion with membrane antigen and subsequent hybridoma development, showed well defined specificity for colon cancer. There was minimal if any evidence of cross reactivity of these antibodies to surrounding normal colonic tissue. These monoclonals, developed from the original Hollinshead tumor antigen, were specifically produced in the GMP format for initiation of approved clinical trials. The lgG1 developed for use in such clinical trials was found to function in the same manner as those antibodies found in the host circulation as a response to administration of the original tumor vaccine; that is the development of a strong ADCC response. The FDA did require defining at which stage of tumor development did the target protein appear. This would then clarify as to when the antibody was to be delivered clinically. It appeared that tumor antigen could be detected in normal appearing colonocytes approximately 6months prior to the appearance of those phenotypic features characterizing the development of the colon cancer lesion.

A detailed analysis of the three major monoclonals we had produced against colon cancer antigen revealed that all had the capabilities of inducing a strong ADCC response. This appeared to be the primary mechanism for targeting tumor rather than induction of cytotoxic T cells. Similarly, these mAbs showed a high degree of specificity and sensitivity for the diagnosis and presence of tumor when employed in a serum ELISA. Using Immunohistochemistry $(\mathrm{IHC})$ to define expression of antigen in the tissue under examination, it was found that those cells that had undergone initial genotypic changes, could for the first time, be clearly identified by antigen expression, even though the phenotypic features of cancer were not yet available for recognition by routine pathologic examination [6] Studies to date have suggested that many of the normal appearing colonocytes adjacent to a malignant lesion, have for the most part undergone genotypic transformation (Fig.1) and are expressing tumor antigen while appearing normal phenotypically.

Obviously during resection of a primary colon lesion by colectomy, it is essential for the pathologist to guarantee that transformed colonocytes if identified, not be left behind in the margins of resection that are to be incorporated in the anastomosis. This now appears to be best achieved by employing IHC with those monoclonal antibodies targeting colon tumor antigen along with the standard H\&E protocol. Normal appearing colonocytes at the margin of a colon cancer resection $\mathrm{H} \& \mathrm{E}$ of normal adjacent to colon ca case \#1 NPC1-C $5 \mathrm{ug} / \mathrm{ml}$ on case \#1 which was read as benign.

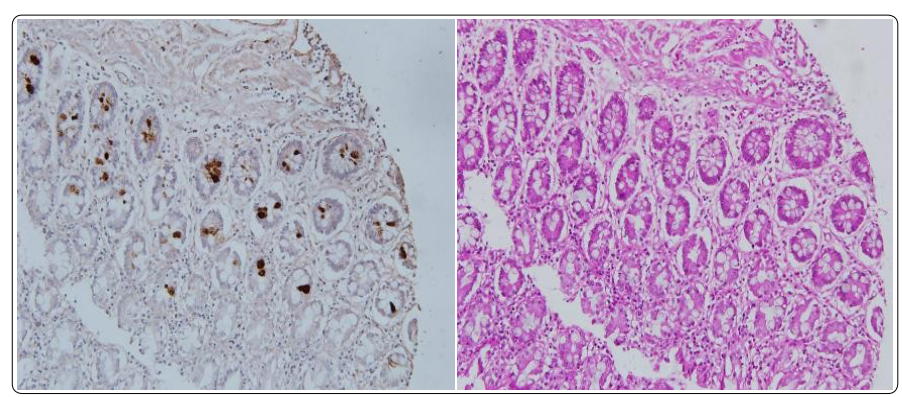

Fig 1. Demonstrates the appearance of colon specific tumor antigen in those normal appearing colonocytes adjacent to the primary malignant colon lesion.

\section{Development of the monoclonal antibodies targeting the tumor antigen}

Tumor antigen structure was analyzed, defined and characterized with the colon cancer mAbs following immunoprecipitation of the pooled allogeneic colon cancer membrane material that had been used as a vaccine in previous early FDA trials. Mass spectroscopy indicated that there were three separate antigens, seen alone and in combination in the various colon cancers, each representing an oncofetal protein needed in the development of the human fetal GI tract. These proteins were usually turned off as the fetus matured by methylation of the gene. In the adult, the onset of malignant transformation of the cell occurs via an oncogenic mutation. This appears to result in a modification of the protein structure through a mutation in the synthetic pathway or possibly thru a post translational modification of the oncofetal protein. The resulting tumor protein was found to be immunogenic and serves to characterize the tumor system in which it is expressed. The tumor cell itself does not have the threshold level of protein present to allow for normal host rejection, the level only present in that amount allowing for immunosurveillance. Effective therapy, especially useful in preventing recurrence post surgery, requires the treating physician to deliver the proper threshold level of antigen at approximately 500-1000 ugms., in the form of a vaccine. Within 4-6 months, high titers of circulating antibody can be detected in the serum. In contrast to this, passive therapy needed in the treatment of advanced metastasis can be achieved by administering the effective product derived from the vaccine, that is the monoclonal antibody. An effective intravenous dose of approximately $4 \mathrm{mg} / \mathrm{kg}$ is needed to achieve a clinical response. This latter procedure is employed in treating those advanced cases where the recurrent tumor has failed all forms of therapy.

The immunogenic proteins that we identified were shown to be related to modified forms of MUC5ac, A33, and CEAcam 5,6 . While our monoclonals clearly define these proteins on Immunohistochemistry, commercial monoclonals used to define the known non modified antigens (oncofetal proteins) failed to recognize expression of the modified antigen in the malignant system and were not capable of inducing any form of therapeutic response.

All of the monoclonals that we have developed fit into a unique class of antibodies that are both diagnostic/ therapeutic $\lg G^{\prime}$ s in solid tumor malignancies as represented by colorectal cancer. To date no other anti-tumor lgG1 monoclonals have been found capable of performing in a similar fashion, as characterized by the results obtained when utilizing the epidermal antibodies targeting epidermal growth factor I and II. All of these products have corresponding targets in normal tissue.

\section{Retrospective Study indicating the value of a Rapid IHC protocol}

With the ability to identify the presence of transforming colonocytes in a field adjacent to a primary colon cancer. It is now possible to reduce if not eliminate a major cause leading to anastomotic tumor recurrence. In a review of those 
colectomy cases that presented post surgery with anastomotic recurrence, we noted that mucosal abnormalities could readily be detected adjacent to the primary lesion. Such changes had gone unrecognized at the time of surgery, when standard histologic procedures were employed. By utilizing monoclonal antibodies (mAbs) that define the presence of tumor immunogenic proteins, we were able to reexamine socalled normal biopsy sites adjacent to the tumor. Here, we demonstrated the presence of altered cellular activity in existing colonocytes that were in the process of transforming to the full malignant state Fig(2). Biopsy taken from margin of resection in patient presenting with anastomotic recurrence.

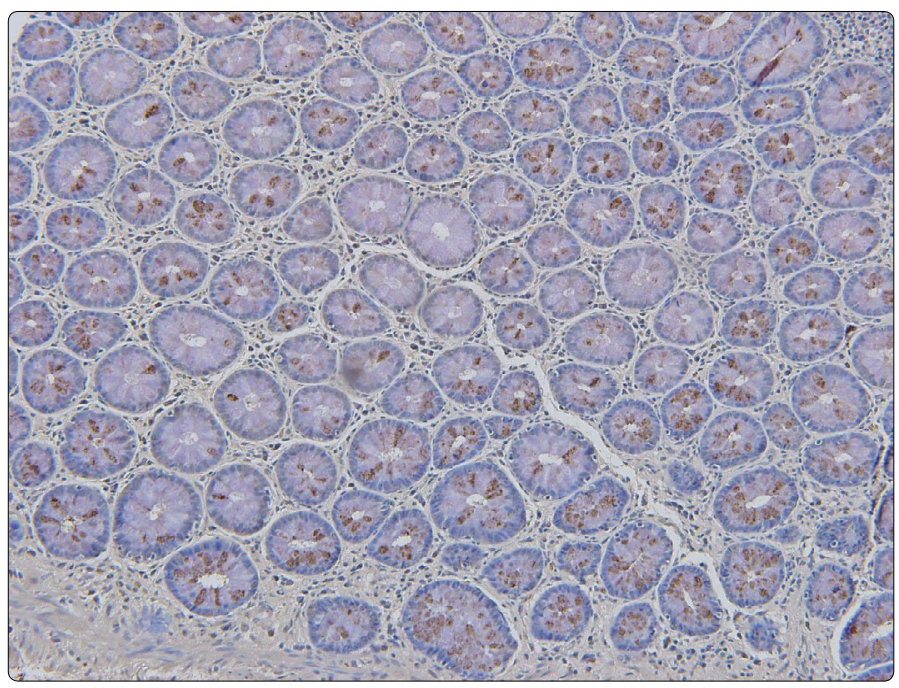

Fig 2. IHC of normal colonocytes adjacent to a primary colonic lesion

\section{Protocol}

In the employment of the proposed IHC procedure, the kit to be employed will be for rapid IHC utilized in the OR so that frozen sections taken from margins of bowel following colectomy can be analyzed by IHC protocol. Positive and negative controls are included to guarantee the relative accuracy of the interpretation offered by the pathologist. If after both $\mathrm{H} \& \mathrm{E}$ and $\mathrm{IHC}$, there is evidence of premalignant cells as well a fully malignant cells present on histologic examination, the surgeon will be notified to make modifications to the extent of his resection in order to guarantee that no potential malignant cells will be left for incorporation into the anastomosis.

To better understand the nature and origin of transforming colonocytes in proximity to a primary colonic lesion, it appears that a field effect may exist around the site of the primary malignancy. Such a field probably represents a site of oncogenic transformation wherein an array of transforming cells develop secondary to the effect of an oncogenic virus or carcinogen. In this field, the initial group of cells that complete the transformation to a fully malignant phenotype, probably suppress surrounding premalignant cells from further transformation on an immunogenic basis. Removal of the primary lesion may eliminate this suppressive effect and allow premalignant cells i.e. colonocytes expressing antigen, to progress to the fully malignant phenotype.
This phenomenon of a field effect with premalignant yet normal appearing cells adjacent to a malignancy has now been recognized with other lesions such as lung cancer as well as having been seen in pancreatic cancer. Kadara et. [7] employed a genetic approach to examine normal cells adjacent to lung cancer in order to define premalignant changes taking place. Transforming cells that were identified were felt to define early premalignant Changes [8-10].

At present, the standard for following patients undergoing anterior resection or any procedure where the margins of resection might be of concern, is the use of endoscopic ultrasound along with colonoscopy In the future, employing Immunhistochemical procedures in the $O R$ at time of evaluation of margins of resection is expected to become a standard. We believe that this will eventually pre-empt the need for the colonoscopy/EUS which defines the lesion as it is developing, rather than preventing its occurrence.

\section{Evaluation and Calculation of Results}

In an attempt to accurately describe the extent of Immunohistochemical staining of a tumor, the degree of IHC staining when present is captured for each analysis. The distance of the tumor to the margin site where biopsies are taken for analysis are recorded both as proximal and distal margins.

The algorithm includes capturing the percentage of tumor cells stained at each intensity level. A semi quantitative intensity scale ranging from 0 for no staining to $3+$ for the most intense staining is used. All of this information can be analyzed separately or used to calculate a variable, more continuous than simply Positive versus Negative, called the $\mathrm{H}$-score. This score is more representative of the staining of the entire tumour on the section. Although given sections may share the same simple intensity score, there clearly is a difference between a $3+$ case with only $10 \%$ of the cells staining as compared to a $3+$ case where greater than $90 \%$ of the cells are staining. This difference is easily picked up using $\mathrm{H}$ - score method. An $\mathrm{H}$-score is typically calculated for staining of each sub-cellular compartment for both normal and tumor cells using the following formula:

$\mathrm{H}$ score $=(\%$ at 0$) * 0+(\%$ at $1+) * 1+(\%$ at $2+) * 2+(\%$ at $3+) * 3-$

Thus the score produces a continuous variable that ranges from 0 to 300 .

\section{Protocol Attachment}

\section{Intended use of the supplied Kit}

The provided kit can be used for a rapid intraoperative immunostaining of the frozen sections of resected colorectal/ pancreatic tumors. This kit provides the following major advantages over existing intraoperative IHC procedures: firstly, the whole staining procedure takes less than 10 minutes; secondly, the included enzyme-labeled antibodies (in conjunction with appropriate substrates) provide a specific and robust brown staining of tumor cells (over light blue 
staining of normal cells), thus making simpler, faster and more confident the following interpretation of stained slides by pathologist.

\section{Reagents and Materials provided}

Acetone

Hematoxylin solution Gill \#2 (Cat.No.GHS-216; Sigma)* PBS

Distilled/deionized water

DAB-PLUS Substrate Kit (Cat.No. 002020; Invitrogen)* Mounting media (water-based/xylene-based)

Cover slip

Xylene (optional) Glass jars

Slide dryer at 37 oC Pipettor

Timer

Light microscope

\section{Staining Protocol}

1. Prepare $6 \mathrm{mkm}$ slides from the freshly isolated, OCTembedded and frozen colon/pancreatic tissues.

2. Fix $20 \mathrm{sec}$ in acetone (RT). Let to dry. Circle border of section spot with fat/permanent marker if necessary.

3. Put into PBS for $20 \mathrm{sec}$. Shake off an excess of PBS.

4. Place on pre-warmed slide dryer at 37C.

5. Add pre-diluted ( $\mathrm{x} 1$ in $\mathrm{PBS}$ ) antibody mix to slides $\left(100 \mathrm{mkl}^{2} 1 \mathrm{~cm}^{2}\right)$ for $3 \mathrm{~min}$.

6. While incubating with antibody, prepare $x 2$ conc.DAB substrate solution $(200 \mathrm{mkl} / \mathrm{slide})$ using $\mathrm{DAB}$ plus kit (Invitrogen). Use one drop of solution \#1 and two drops of solutions \#2 and \#3 per each $\mathrm{ml}$ of $\mathrm{H}_{2} \mathrm{O}$.

7. Shake off antibody mix then rinse consequently in two beakers with $100 \mathrm{ml} \mathrm{PBS}(5 \mathrm{sec} \times 2)$.

8. Add DAB substrate solution (p.6) for $1.5 \mathrm{~min}$.

9. Rinse in PBS.

10. Dip once (5sec.) in Gill \#2 hematoxylin solution.

11. Wash briefly to develop blue color in $\mathrm{NH} 4 \mathrm{OH}(0.4 \%)$ in tap water $(500 \mathrm{ml})$. Put into $\mathrm{dH} 2 \mathrm{O}$.

12. Mount using water-based mounting medium.

13. Analyze under light microscope (x40-x400).

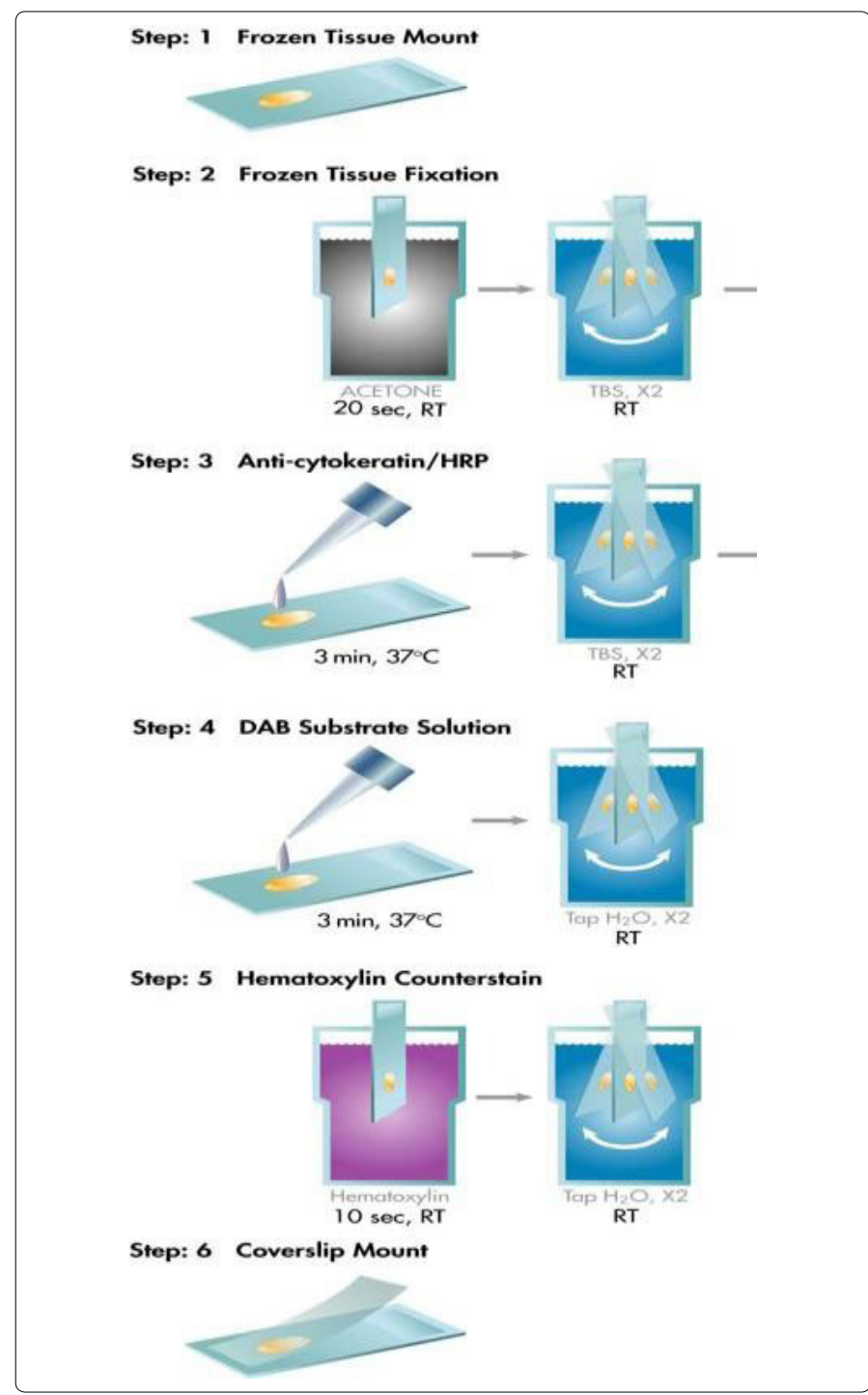

Figure 3. Rapid IHC protocol at glance

\section{Expected Results}

The supplied antibodies used in this kit react with at least two human carcinoembryonic antigen related molecules (CEACAM5/6) and several tumor- specific mucins (MUC5AC, MUC2 etc). The antibodies can be used for rapid detection of primary and metastatic colon/pancreatic tumors in frozen sections. The staining intensity usually reflects the effects of tissue preparation as well as antigen concentration.

Although developed for rapid detection of colorectal/ pancreatic tumors and their margins in frozen sections, this kit is capable to detect additionally several other epithelial carcinomas (lung, ovary) if used in standard IHC procedure of paraffin sections**. The latter assay can be performed without antigen retrieval step. An intense staining indicates a relatively higher abundance of tumor- associated antigens, while a lighter staining is indicative of a lower antigen concentration in given specimen.

* Supplier of this reagent is shown as an example. However, incubation time should be adjusted if reagent from another supplier is used.

** Cocktail should be diluted 80 times (x0.25). 


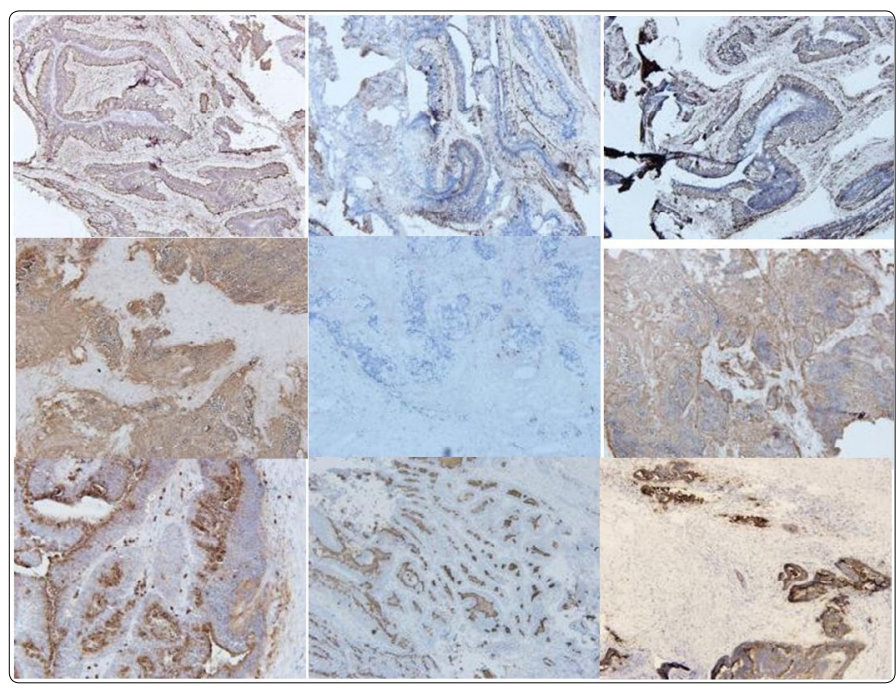

Fig 4. Comparative staining of 3 colon cancer tissues

\section{References}

1. Arlen M, Hollinshead AC, Tsang KY. Identification and Characterization of a Colon TAA. Annals N.Y.Academy of Science. 1993.

2. Tsang KY, Bishop $E$, Johnson $S$, Chiofalo $M$, LaVia $M$, Arlen $M$. Characterization of murine monoclonal antibodies reactive to human colon carcinoma-associated antigens. abst. Fifth Internat.Symp on Rapid Methods and Automation in Microbiology and Immunology -Ital. Soc, Microbiology 1987.
3. Arlen M, Tsang KY. The Nature of the Monoclonal Antibodies Derived from Immunogenic Membrane Antigen of Human Colon Carcinoma Origin. J.Tumor Marker Oncology. 1990; 5: 313-319.

4. Arlen M, Tsang A, Wang T. Immunotherapy of Colon Cancer Using Chimeric mAb31. Crit. Reviews in Immunology. 1998; 18(1-2): 133-138.

5. Hollinshead AC, Stewart THM, Elias G, Arlen M. Co-assesment of Serum Epitope Antibodies, Cell Mediated Immunity and Survival in Colon Cancer Patients on TAA Specific Active Immunotherapy. Proc. Sixth Int. Conf. on Adjuvant Therapy of Cancer. W.B.Saunders. Section IX Colorectal Cancer, 454-468, 1990.

6. Arlen $M$, Arlen $P$, Coppa $E$, et al. Identification of the Oncofetal Tumor Antigens that Characterize Colorectal Carcinoma for use in Diagnosis and Therapy. J. Gastroent.Oncol. 2013; 10: 156-169.

7. Arlen M, Tsang KY. The Nature of the Monoclonal Antibodies Derived from Immunogenic Membrane Antigen of Human Colon Carcinoma Origin. J.Tumor Marker Oncology. 5:313-319,1990

8. Arlen M, Tsang A, Wang T. Immunotherapy of Colon Cancer Using Chimeric mAb 31. Crit. Reviews in Immunology. 18:133-138,1998

9. Hollinshead AC, Stewart THM, Elias G, Arlen M. Co-assesment of Serum Epitope Antibodies,Cell Mediated Immunity and Survival in Colon Cancer Patients on TAA Specific Active Immunotherapy. Proc. Sixth Int. Conf. on Adjuvant Therapy of Cancer. W.B.Saunders. Section IX Colorectal Cancer,454-468, 1990

10. Arlen $M$, Arlen $P$, Coppa $E$, et al. Identification of the Oncofetal Tumor Antigens that Characterize Colorectal Carcinoma for use in Diagnosis and Therapy J. Gastroent.Oncol 2013 10;156-169 